\title{
Management of Nasal Dermoid Sinus Cyst by Modified Bipedicle Advancement Flap Technique
}

${ }^{1}$ Chidananda Ramappa Devasamudra, ${ }^{2}$ Niveditha J Sagar

\begin{abstract}
Midline congenital nasal lesions are rarely encountered in adults. Nasal dermoid sinus cysts (NDSC) account for 1 to $3 \%$ of dermoid cysts overall and 11 to $12 \%$ of head and neck dermoids. Most lesions are diagnosed within the first 3 years of life but in some cases the diagnosis can be delayed. Nasal dermoid sinus cysts are unsightly, prone to infection. Treatment is complete surgical excision. We are presenting a case of 21-year-old man with a NDSC who presented with soft tissue hump over the bony nasal dorsum, recurrent infection and discharge from the two sinus openings over the bony nasal dorsum. We review the embryology of nasal dermoid sinus cysts, discuss their presentation, evaluation and management in adults. Radiological investigations like computed tomography (CT) scan, magnetic resonance imaging (MRI) are essential to know the extension of the sinus tract and CNS involvement. Complete surgical excision and reconstruction of the defect over the bony nasal dorsum by modified bipedicle advancement flap technique will help to give esthetically good looking nose.
\end{abstract}

Keywords: Cyst, Dermoid, Excision, Flap, Modified bipedicle advancement flap technique, Nasal, Reconstruction.

Abbreviations: CNS: Central nervous system, CT: Computed tomography, MRI: Magnetic resonant imaging, NDSC: Nasal dermoid sinus cyst, PNS: Para nasal sinus.

How to cite this article: Devasamudra CR, Sagar NJ. Management of Nasal Dermoid Sinus Cyst by Modified Bipedicle Advancement Flap Technique. Clin Rhinol An Int $\mathrm{J}$ 2015;8(3):115-120.

\section{Source of support: Nil}

Conflict of interest: None

\section{INTRODUCTION}

Midline congenital lesions of the nose are rare congenital anomalies. The differential diagnosis of midline nasal masses includes inflammatory lesions, post-traumatic deformities, benign neoplasms, malignant neoplasms,

\footnotetext{
${ }^{1,2}$ Assistant Professor

1,2Department of ENT, Head and Neck, Sapthagiri Institute of Medical Sciences and Research Center, Bengaluru Karnataka, India
}

Corresponding Author: Chidananda Ramappa Devasamudra Assistant Professor, Department of ENT, Head and Neck Sapthagiri Institute of Medical Sciences and Research Center Bengaluru, Karnataka, India, Phone: 8971611749, e-mail: devasamudra@gmail.com and vascular masses. ${ }^{1,2}$ Gliomas, encephaloceles and nasal dermoid sinus cysts (NDSC) are the main part of the congenital midline lesions of the nose. ${ }^{2,3}$ Nasal dermoid sinus cysts account for 1 to $3 \%$ of dermoid cysts overall and 11 to $12 \%$ of head and neck dermoids. ${ }^{4,5}$

Nasal dermoids typically present at birth or shortly thereafter. Patients present with a nasal mass, a pit, or a fistulous tract. Hair or sebaceous material may protrude from the pit and some patients present with recurrent drainage.

Most infections are limited to the sinus and cyst, but periorbital cellulitis and osteomyelitis have been reported. ${ }^{6}$ These occur in the midline, anywhere from the columella to the glabella. Unlike encephaloceles, these masses are firm to solid, do not enlarge with crying, and do not transilluminate. Most dermoids terminate in a subcutaneous tract, but penetration deep to the nasal bones occurs in up to $45 \%$ of patients, with intracranial extension noted in 25 to $30 \%$ of cases. ${ }^{7}$

Early diagnosis is made in the first 3 years after birth in most cases. But in some cases the diagnosis may be prolonged. ${ }^{2,3,8}$ We are presenting a 21-year-old adult with a rare congenital nasal midline lesion and its surgical treatment with a review of the literature.

\section{CASE REPORT}

A 21-year-old male patient presented with chronically draining two sinus openings over the bony dorsum of the nose since birth and with recurrent nasal midline infection (Fig. 1). This patient is also complains of hump over the nose which is causing disfigurement of nose (Fig. 2). He had no other complaints. He had no specific history within his family and no maxillofacial trauma. On external nose examination, there was soft tissue hump over the bony nasal dorsum, two draining sinus openings (big opening with dark colored hairs on the midline over the bony dorsum and small opening on the left side of the bony nasal wall, $5 \mathrm{~mm}$ inferolateral to the big opening) on the skin of the bony dorsum and thick dark colored hairs around the sinus openings (Fig. 1). On anterior rhinoscopy examination, nasal mucous membrane nasal septum and turbinates were normal. Interestingly, this patient had unilateral preauricular sinus on the left side (Fig. 2) and hyper pigmented skin around the left 
periorbital area. No other findings to correlate Syndromic association and needs further evaluation, studies to do so.

In the computed tomography (CT) scan, sinus tract was extending posteriorly involving the superficial surface of nasal bones and magnetic resonance imaging (MRI) scan showed there was no intracranial extension (Figs 3 and 4).

We performed dermoid sinus cyst excision and reconstruction of the nasal dorsum by modified bipedicle advancement flap (dorsal approach rhinoplasty) under general anesthesia. Methylene blue dye is injected to the midline sinus opening (Fig. 5) and the dye came out through the adjacent small sinus opening. So it confirms the connectivity of both sinus openings. Rhomboid incision is given over the dorsum of the nose which includes both sinus openings. The incision is extended at the upper and lower corner of rhomboid incision for advancement flap procedure (Fig. 6).

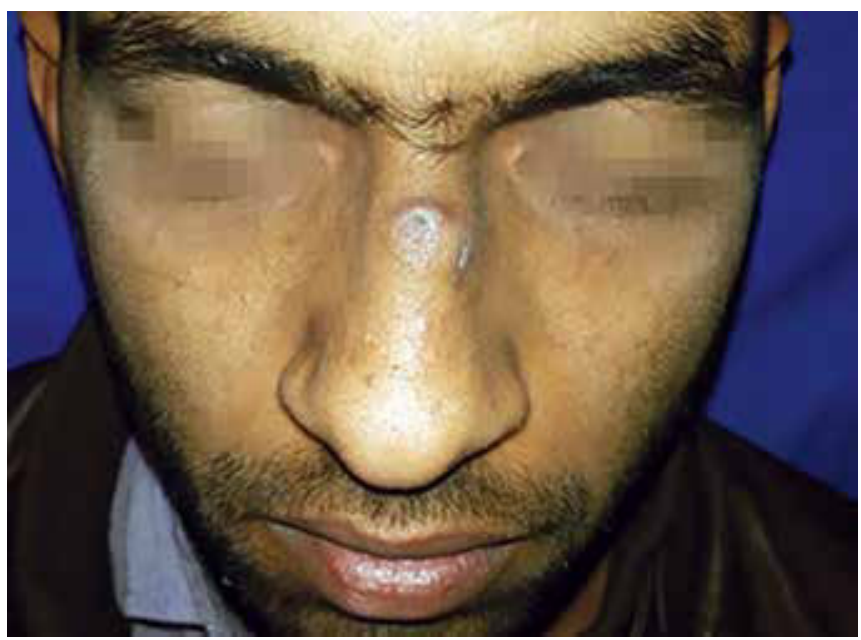

Fig. 1: Nasal dermoid sinus cyst over bony nasal dorsum

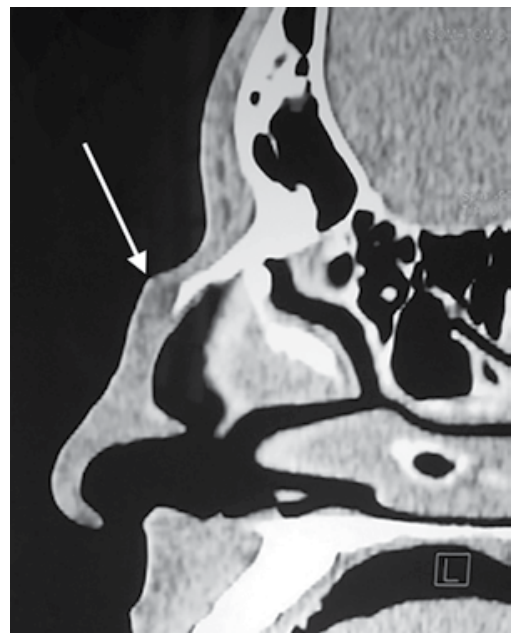

Fig. 3: Computed tomography scan nose PNS (sagittal view) showing extent of NDSC
The sinus tract was extending toward posteriorly in between nasal bones. The sinus tract was completely removed (Fig. 7). While removal of the tract, we could see the hairs in the tract (Fig. 8). Surgical wound defect measuring about $1 \times 1 \mathrm{~cm}$ is created after sinus excision. To close the defect, modified bipedicle advancement flap technique is used (Fig. 9). Full thickness soft tissue flap around the defect, underneath the dorsum of the nose is elevated to pull the bipedicle flap and approximate the surgical wound (Fig. 9).

Surgical wound is sutured in two layers. 3-0 vicryl with reverse cutting needle is used to suture the soft tissue flap (buried sutures). 5-0 prolene with reverse cutting needle is used to suture the skin (simple sutures). Surgical wound defect is closed from rhomboid area to reverse ' $Z$ ' shaped suture line (Fig. 10). Dog ear deformity is created at the upper end of advancement flap (Fig. 11) because of the more mobile skin over the bony dorsum,

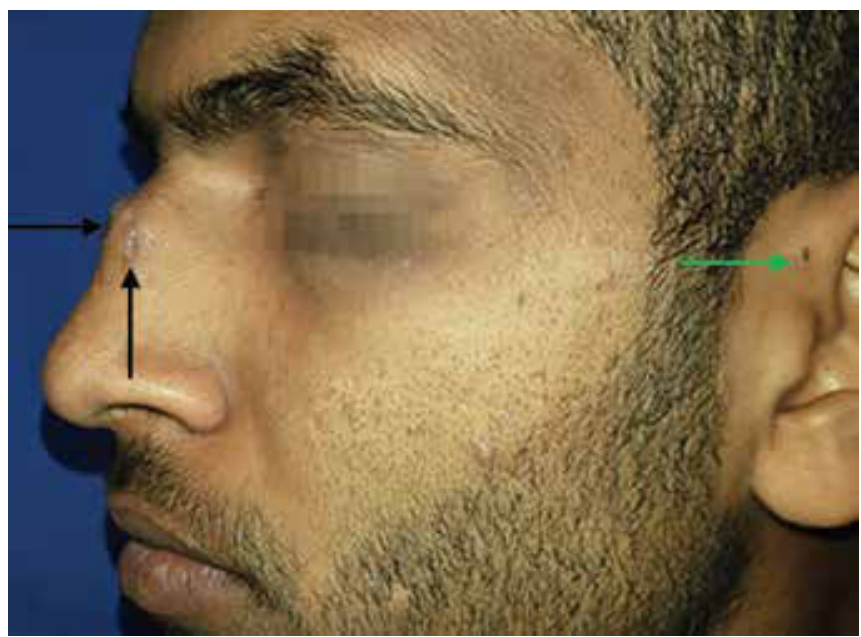

Fig. 2: Lateral view showing hump over bony nasal dorsum, two openings (black arrows) of NDSC. Preauricular sinus opening (green arrow) is also seen

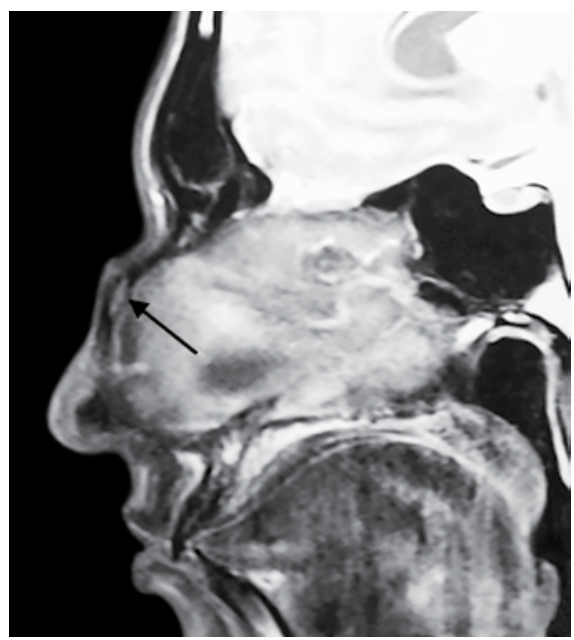

Fig. 4: Magnetic resonance imaging scan showing NDSC with no intracranial extension 


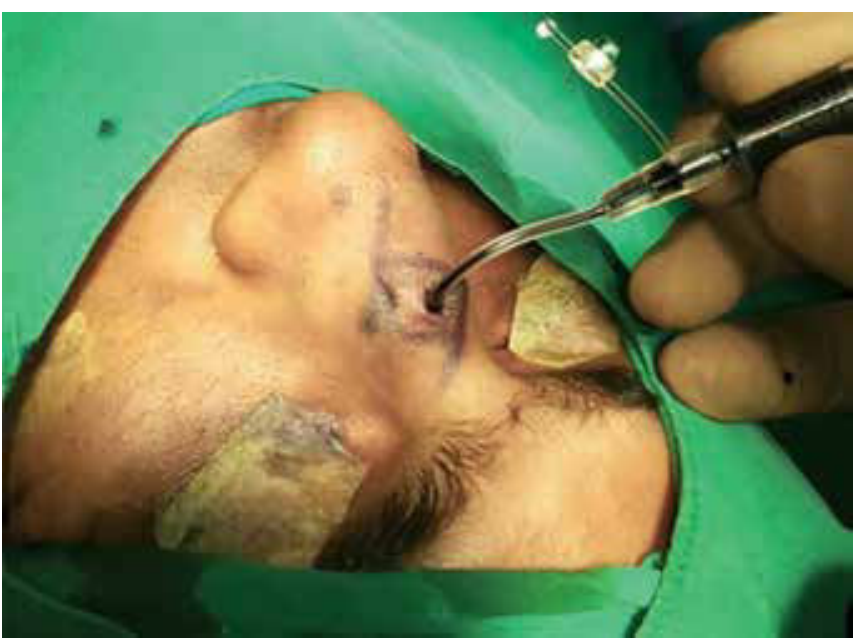

Fig. 5: Methylene blue dye injected, to track the sinus

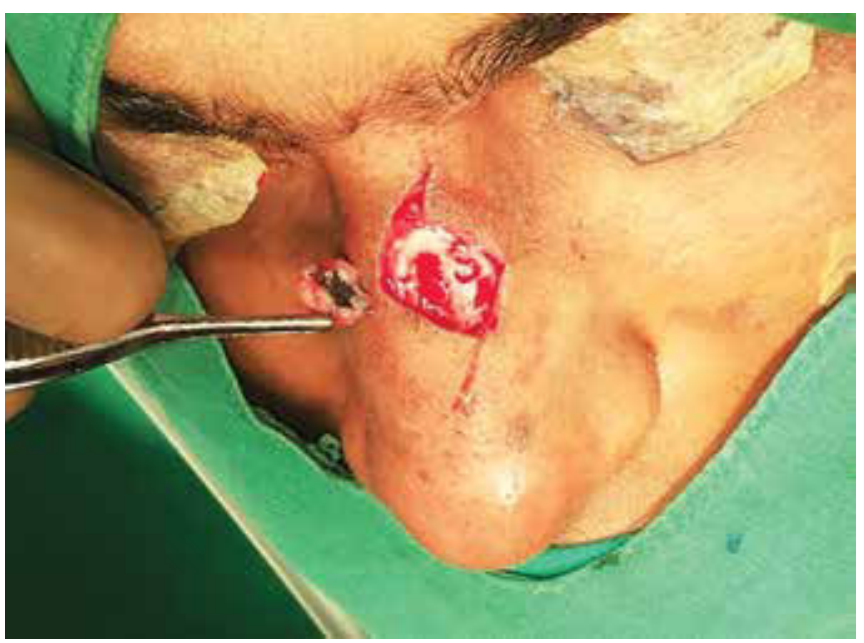

Fig. 7: Excised specimen, surgical wound defect

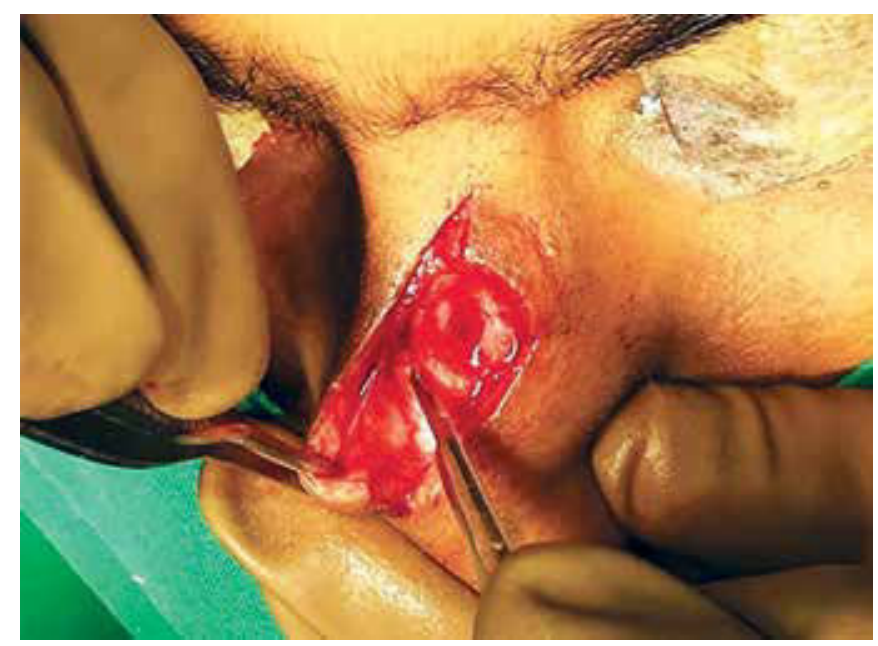

Fig. 9: Flap elevation underneath the surgical wound to advance and approximate the wound (for modified bipedicle advancement flap)

which is corrected by excising a small triangular skin (Fig. 11). Interestingly, we could not get dog ear deformity at the lower end of the advancement flap, because the skin over the supra tip area is adherent to underlying

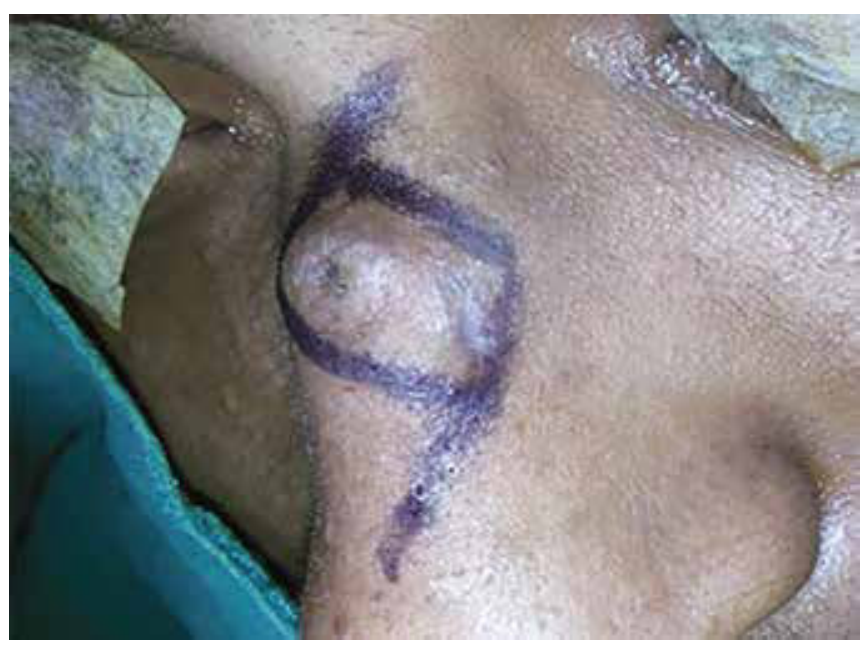

Fig. 6: Skin marking for incision, complete excision and reconstruction of surgical wound

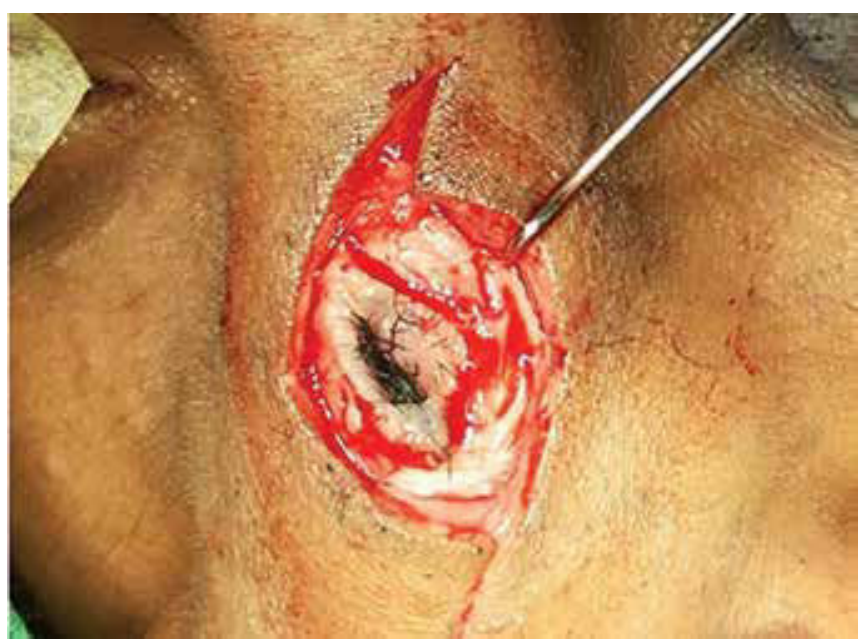

Fig. 8: Sinus tract showing thick dark colored hairs

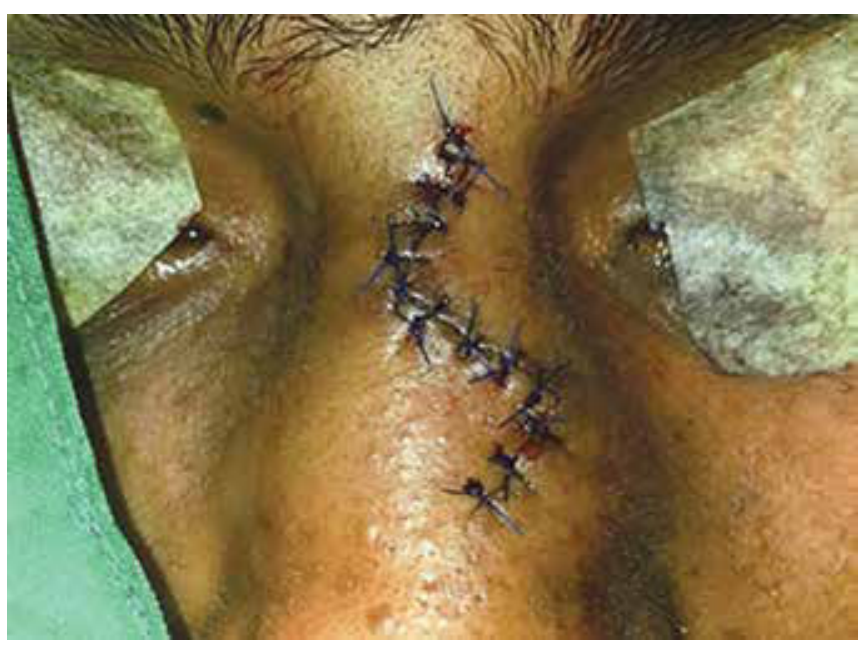

Fig. 10: Surgical wound closure after modified bipedicle advancement flap technique

structures. Postoperative period was uneventful and the patient was discharged next postoperative day. The postoperative pathology report was epidermal inclusion cyst. The patient had no complaints in the follow-up visits and the esthetic results were satisfying (Fig. 12). 


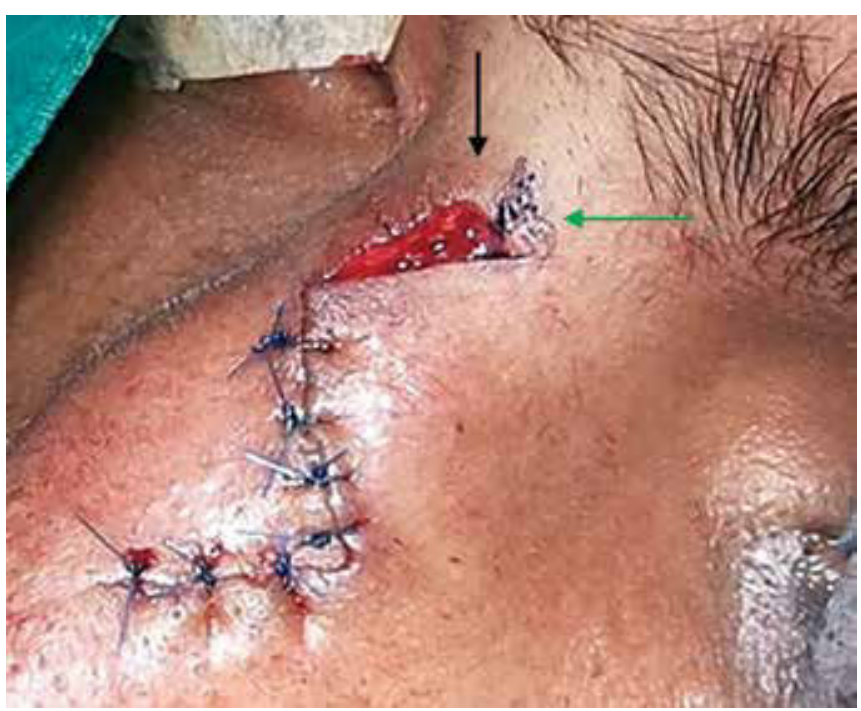

Fig. 11: Dog ear deformity (black arrow), triangular skin marking (green arrow) to correct dog ear deformity

\section{DISCUSSION}

In 1817, Cruvier first described a patient with a midline nasal pit with a small hair growing from it. ${ }^{9}$ Many terms have been used to describe these lesions, including dermal cyst, dermoid, dermoid cyst, and dermoid sinus cyst. In 1982, Sessions introduced the term NDSC to refer to such lesions with a sinus tract, whereas nasal dermoid cysts do not. Nasal dermoid sinus cysts contain ectoderm (stratified squamous epithelium) and mesoderm (adnexal structures, such as sebaceous glands and sweat glands).

Dermoids also can occur at other sites, including the forehead, anterior fontanelle, orbit and periorbital areas, tongue and neck. Nasal dermoid sinus cysts account for 1 to $3 \%$ of dermoid cysts overall and 11 to $12 \%$ of head and neck dermoids. ${ }^{4,5}$

The most popular theory is that the anterior neuropore fails to close properly in the region of the fonticulus frontalis or foramen cecum. Thus, ectoderm can become trapped outside the closing neuropore, and if it remains adherent to the dura as it retracts into the cranium, it can result in a sinus tract connecting the NDSC to the intracranial space. ${ }^{10}$ Grumwald, who in 1910 implicated inadequate obliteration of the prenasal space of its dural connection, ${ }^{11}$ put another forth. This too involves the abnormal closure of the foramen cecum with epithelium trapped anywhere along the tract, forming the cyst or sinus tract. Another theory says that the middle laver of the fetal trilaminar nasal septum is composed of dura, which normally obliterates. Failure to do so results in NDSC..$^{13}$ Other theories include aberrant development of skin appendages, ${ }^{12}$ inclusions in facial clefts, and simple inclusion cysts. ${ }^{12,13}$

Nasal dermoid sinus cysts are typically seen as midline masses. They usually have a sinus opening in the nasal dorsum. ${ }^{14,15}$ Intermittent secretion of sebaceous

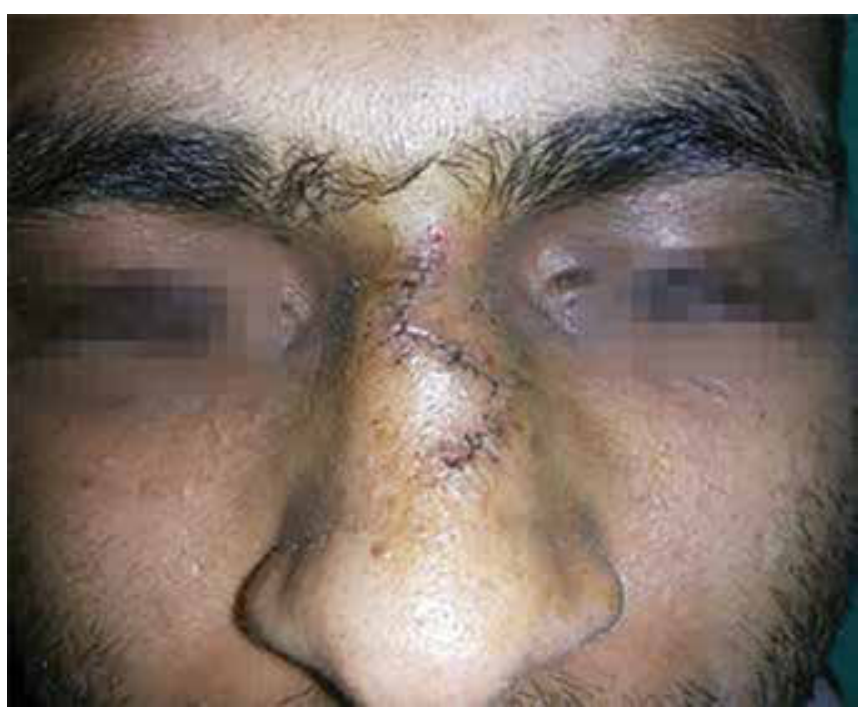

Fig. 12: Postoperative picture after 10 days

material and recurrent infections are seen frequently. The hair outgoing from the opening is pathognomonic for the NDSC but is found in less than half of the patients.,14 Nasal dermoid sinus cyst is seen sporadically but familial cases have been reported in the literature. ${ }^{1,4}$ There is no association of a syndrome with the formation of the NDSC.

Because of the inherent risk of possible intracranial connection, these lesions should never be biopsied initially. Radiographic examination is necessary to assess the depth of involvement and for the presence of extension into the intracranial space. Computed tomography scans demonstrate associated bony anomalies well and may show the classic findings occasionally seen on plain films of swelling or bifidity of the bony nasal septum or widening of the nasal vault. A patent foramen ovale or bifid crista galli are suggestive of an intracranial mass. Magnetic resonance imaging is the imaging study of choice to determine the depth of the tract and is superior to $\mathrm{CT}$ in determining intracranial involvement. Intraosseous dermoids may be difficult to visualize, however, and normal fatty deposits associated with development of the frontal sinuses and nasal bones can be read as false positives. ${ }^{16}$ The treatment of the NDSC is surgical excision..$^{3-5,14}$ The most favorable technique is dorsal nasal approach with Modified bipedicle advancement flap like in our case. The reasons for choosing this technique are exposure, good esthetic results and allowing the reconstruction of the nasal dorsum. The formation of the surgery depends on the lesion's localization and extension.

\section{Modified Bipedicle Advancement Flap}

Reconstruction of the defects in the nasal dorsum after the excision of lesions like dermoid or malignancy is difficult task, because of the complexity of the nasal anatomy, curvatures and possibility of ugly external scar. 
There are many different techniques to reconstruct dorsal nasal defects depending on the location, size, shape and depth of the defect. For example, bilobed rotation flap for small defect over lateral wall of the nose or forehead flap to close large defect over the supra tip area.

In the routine bipedicle advancement flap technique, the defect is closed by elevating the rectangular flap on both sides. After pathological lesion extirpation, the length and width of the primary defect are measured. After under-mining, the flap is incised linearly at a distance equal to or slightly greater than the width of the wound, corresponding to the amount of tissue movement needed to close the primary defect. The length of this incision corresponds to the long axis of the original defect and parallels the wound. The flap is then further undermined, elevated and advanced. This enables closure of the original defect primarily. The resultant four dog ears created at the lateral wound margins can then be excised and sutured in the usual fashion (Fig. 11).

In this case, we modified the bipedicle advancement flap technique to reconstruct the rhomboid surgical defect over the bony nasal dorsum. The defect was more toward left lateral wall of the nose, because this patient had two sinus openings, large one at the midline and the other small opening $5 \mathrm{~mm}$ inferolateral to large opening over the bony dorsum. After excision of NDSC, we created rhomboid skin defect over the bony nasal dorsum (more toward left lateral wall of the nose). To get single reverse $Z$ shaped suture line we have to elevate the upper flap more toward the bony nasal dorsumon the right side and lower flap more toward the lateral wall of the nose on the left side. By doing this, we can avoid routine ' $\mathrm{H}$ ' shaped scar of bipedicle advancement flap technique. The vital areas like medial canthus on the left side and Skin over the Supra tip area are not advanced in closure of the defect, so it preserves the facial esthetics. And we get only one dog ear deformity at the level of nasion which is corrected by triangular skin excision (But in routine bipedicle advancement flap technique, we get four dog ear deformities). This modification will help, to give more acceptable single linear scar over the nasal dorsum.

\section{Dorsal Approach Rhinoplasty ${ }^{17}$}

In this case, dorsal approach rhinoplasty is done to reduce the hump over the nose. Rhinoplasty can be done by various surgical approaches like:

- Closed (internal) approach

- Open (external) approach

- Dorsal approach

- Combination of any two approaches.

In closed approach, all the incisions will be inside the nasal cavity. Patient will not get any external scar but the tissue exposure will be limited. Procedures like Tipplasty, osteotomies can be done by closed approach and it needs more surgical expertize.

In open approach, columellar incision (inverted $\mathrm{V}$ shaped, step or Z shaped) will give rise to visible scar. All other incisions will be hidden in nasal vestibule and nasal cavity. The advantages of this approach will be more exposure of nasal framework. So, under vision, surgeon can do procedures like humpectomy, cartilage suturing. The disadvantages are, this approach is time consuming and more surgical trauma to the patient.

In dorsal approach rhinoplasty, the incisions will be over the nasal dorsum. As in this case, the incision is over the dorsum of the nose. The congenital lesions like NDSC, neurofibromatosis and skin lesions can be managed by dorsal approach. The advantages are, more exposure and procedures like skin resection can be done. The disadvantage is, the patient may get ugly external scar.

In some rare circumstances, surgeons may use both open and dorsal approach to achieve the required results. This will be the combination approach rhinoplasty.

\section{CONCLUSION}

Nasal dermoid sinus cyst is a rare midline nasal swelling, which will lead to nasal disfigurement and chronic discharge from the sinus opening which is embracing to the patient. Radiological investigations (both CT and MRI) are essential to know the extension of sinus and involvement of CNS. Nasal dermoid sinus cyst over the bony dorsum is managed by complete removal of the sinus. Surgical defect is closed by modified bipedicle advancement flap technique to give minimal scar and preservation of facial esthetics.

\section{REFERENCES}

1. Zapata S, Kearns DB. Nasal dermoids. Curr Opin Otolaryngol Head Neck Surg 2006;14:406-411.

2. Hanikeri M, Waterhouse N, Kirkpatrick N, et al. The management of midline transcranial nasal dermoid sinus cysts. Br J Plast Surg 2005;58:1043-1050.

3. Post G, McMains KC, Kountakis SE. Adult nasal dermoid sinus cyst. Am J Otolaryngol 2005;26:403-405.

4. Rahbar R, Shah P, Mulliken JB, et al. The presentation and management of nasal dermoid: a 30-year experience. Arch Otolaryngol Head Neck Surg 2003;129:464-471.

5. Rohrich RJ, Lowe JB, Schwartz MR. The role of open rhinoplasty in the management of nasal dermoid cysts. Plast Reconstr Surg 1999;104:2163-2167.

6. Pratt L. Midline cysts of the nasal dorsum: embryologic origin and treatment. Laryngoscope 1965;75:968-980.

7. Fornadly J, Tami T. The use of MRI in the diagnosis of nasal dermoid sinus cyst. Otolaryngol Head neck Surg 1989;101: 397-398.

8. Yavuzer R, Bier U, Jackson IT. Be careful: it might be a nasal dermoid cyst. Plast Reconstr Surg 1999;103:2082-2083.

9. Sessions R. Nasal dermoid sinuses: new concepts and explanations. Laryngoscope 1982;92(Suppl 29):1-28. 
10. Hughes G, Sharpino G, I lunt W, et al. Management of the congenital midline nasal mass: a review. Head and Neck Surg 1980;2:222-233.

11. Sessions R, Hudkins C. Congenital anomalies of the nose. In Bailey B: Head and Neck Surgery-Otolaryngology. Philadelphia: JB Lipincott; 1993. p. 1.

12. Hoshaw T, Walike J. Dermoid cysts of the nose. Arch Otolaryngol Head Neck Surg 1971;93:487-491.

13. Littlewood A. Congenital anomalies of the nose: their embryology, diagnosis and management. 2nd ed. Am Acad Otolaryngol-Head and NeckSurg Foundation, Inc. 1987;2:25-83.
14. Bilkay U, Gundogan H, Ozek C, et al. Nasal dermoid sinus cysts and the role of open rhinoplasty. Ann Plast Surg 2001 p. 478-414.

15. Sreetharan V, Kangesu L, Sommerlad BC. Atypical congenital dermoids of the face: a 25-year experience. J Plast Reconstr Aesthet Surg 2007;60:1025-1029.

16. Barkovich A, Vandermarck P, Edwards M, et al. Congenital nasal masses: CT and MR imaging features in 16 cases. Am J Neuroradiol 1991;12:105-116.

17. Dubeta KR, et al. Dorsal approach rhinoplasty: otorhinolaryngology clinics. Int J 2013 Jan-Apr;5(1):1-23. 